\title{
Itch in psoriasis: epidemiology, clinical aspects and treatment options
}

\section{F Prignano \\ F Ricceri \\ L Pescitelli \\ $\mathrm{T}$ Lotti}

Department of Dermatological Sciences, University of Florence, Florence, Italy
Correspondence: Francesca Prignano Department of Dermatological Sciences, University of Florence, Villa Basilewsky, via Lorenzo II Magnifico n I04, 50129 Florence, Italy

Tel +55 $626448 \mathrm{I}$

Email francesca.prignano@unifi.it
Background: Pruritus is an important symptom in psoriasis vulgaris, may be severe and seriously affect the quality of life of patients, but published data on its frequency and characteristics are limited.

Objective: The study objective was to characterize the prevalence of itch in psoriatic patients and the effect of treatment modalities by using a comprehensive itch questionnaire of own design.

Methods: A structured itch questionnaire was given to 90 patients with moderate to severe chronic-plaque psoriasis selected consecutively from the patients visiting the Department of Dermatology of the University of Florence. The questionnaire concerned the areas involved psoriasis and pruritus, the pruritus characteristics, the worsening and relieving factors and treatment modalities. Itch intensity was reflected by a 10 point visual analog scale (VAS) and the degree of symptoms discriminated between mild (1-3), moderate (4-7) and severe (8-10).

Results: Almost $85 \%$ of psoriatic patients suffered from itching; the frequency of pruritus was daily and mean intensity by VAS scale was moderate. Presence and intensity of pruritus and body mass index (BMI) were correlated. $40 \%$ of patients with pruritus were overweight (BMI $>25<30$ ) and $10 \%$ obese $(\mathrm{BMI}>30)$. Almost all patients appeared unsatisfied with the available treatment modalities for pruritus in psoriasis. Emollients, topical steroids and calcipotriol cream could relieve pruritus but their effect was temporary. Among the antipsoriatic therapies, phototherapy with narrow band ultraviolet B (nb-UVB) was the most effective treatment in reducing pruritus. Biological therapies, mainly etanercept and efalizumab, proved useful in its control.

Conclusions: The questionnaire was a useful tool to characterize itch, and the results might help us to better understand pruritus in psoriasis. The results confirmed the need for a global study of psoriasis with regard to both the cutaneous manifestations and the itch symptom.

Keywords: itch, psoriasis, pruritus, epidemiology, phototherapy, etanercept, efalizumab

\section{Introduction}

Itch (pruritus) is a common symptom of many dermatological and systemic diseases. Although very common, itch is very hard to describe. According to Bernhard: "itching is a sensation that, if sufficently strong, will provoke either conscious or reflex scratching or the desire to scratch". ${ }^{1}$ It consists of multidimensional phenomena with sensory discriminative, cognitive, evaluative and motivational components. Itch has similarities with pain, but in contrast to pain itch induces scratch cycles, which combine elements of pleasure with pain. ${ }^{2}$

Severe itch can severely affect quality of life (eg, sleep deprivation) and can have serious psychological implications (including temptation to suicide). Mild to severe pruritus is a common symptom in numerous inflammatory skin disorders, including atopic dermatitis, eczema or lichen planus. Pruritus is an important symptom also in psoriasis vulgaris, but published data on both its prevalence and characteristics are limited. Its prevalence in different parts of the world ranges fromm $64 \%$ to $84 \% .^{3,4}$ 
According to Reich et al pruritus intensity in psoriatic patients is significantly correlated with quality of life impairment, level of stigmatization and severity of depressive symptoms. ${ }^{5}$

The objective of our study was to characterize the prevalence of the itch in psoriatic patients and the effect of treatment modalities by using a comprehensive itch questionnaire.

\section{Methods}

This study was conducted between October 2007 and March 2008 in the Department of Dermatology of the University of Florence.

A structured itch questionnaire of our own design (Table 1) was given to 90 patients with moderate to severe chronic-plaque psoriasis. Patients were selected consecutively from those visiting the Department in this period. All patients who had other dermatological or general illnesses (such as renal or hepatic diseases) causing pruritus were excluded from the study.

The 90 study patients ( 45 females and 45 males; age range 21-84 years) were divided into 3 groups according to the treatment. Thirty patients were on narrow-band ultraviolet B (nb-UVB) treatment, 30 on topical or systemic traditional treatments, and 30 underwent biological treatment with anti-tumor necrosis factor- $\alpha$ (anti-TNF- $\alpha$ ) or anti-CD11a monoclonal antibody.

Average disease duration was 1 to 65 years. Signs and symptoms of psoriatic arthritis were shown by $15 \%$ of patients.

After a brief explanation of the research project, patients were invited to fill in a questionnaire on pruritus and psoriasis; the questionnaire took approximately 10 minutes to complete. No patients refused to respond to the questionnaire.

The questionnaire asked about age, sex, height and weight, job category, and marital status. It concerned the areas affected by psoriasis and pruritus, pruritus characteristics, worsening and relieving factors, and treatment modalities. A visual analog scale (VAS) of 0 to 10 was used to score pruritus as mild (1-3), moderate (4-7) and severe (8-10).

Statistical analyses were done using the SPSS program. Simple descriptions and frequencies of various factors were tabulated. Associations between factors were determined

Table I Questionnaire

\begin{tabular}{|c|c|c|c|}
\hline \multicolumn{4}{|l|}{ Demographic variables } \\
\hline \multicolumn{4}{|l|}{ Age } \\
\hline \multicolumn{4}{|l|}{ Sex } \\
\hline \multicolumn{4}{|l|}{ Height and weight } \\
\hline \multicolumn{4}{|l|}{ Marital status } \\
\hline \multicolumn{4}{|l|}{ Education } \\
\hline \multicolumn{4}{|l|}{ Clinical variables } \\
\hline \multicolumn{4}{|l|}{ Age at diagnosis } \\
\hline Type of psoriasis & \multicolumn{3}{|c|}{${ }^{\circ}$ Vulgaris ${ }^{\circ}$ Pustulous ${ }^{\circ}$ Erytrodermic ${ }^{\circ}$ Artropatic } \\
\hline \multicolumn{4}{|l|}{ PASI } \\
\hline Previous treatments & \multicolumn{3}{|c|}{${ }^{\circ}$ Topical ${ }^{\circ}$ Systemic (CsA, MTX, Retinoids, PUVA, nb-UVB) } \\
\hline \multicolumn{4}{|l|}{ Stress factors } \\
\hline \multicolumn{4}{|l|}{ Pruritus variables } \\
\hline Presence of pruritus & ${ }^{\circ}$ Yes & & ${ }^{\circ} \mathrm{No}$ \\
\hline \multicolumn{4}{|l|}{ Sites of pruritus } \\
\hline Intensity of pruritus (VAS) & 'Mild (I-3) & ${ }^{\circ}$ Moderate (4-7) & ${ }^{\circ}$ Severe $(8-10)$ \\
\hline Frequency of pruritus & ○Seldom & ${ }^{\circ}$ Often & ${ }^{\circ}$ Always \\
\hline During the night & ${ }^{\circ}$ Yes & & ${ }^{\circ} \mathrm{No}$ \\
\hline Signs of scratching & ${ }^{\circ}$ Yes & & ${ }^{\circ} \mathrm{No}$ \\
\hline \multicolumn{4}{|c|}{ Modalities and efficacy of treatments } \\
\hline \multicolumn{4}{|l|}{ Antipruritus therapies } \\
\hline Antipsoriatic therapies & & & \\
\hline
\end{tabular}

Abbreviations: PASI, Psoriasis Area and Severity Index;VAS, visual analog scale; CsA, cyclosporin-A; MTX, methotrexate; PUVA, psoralen + ultraviolet-A; nb-UVB, narrow-band ultraviolet B. 
using a chi-square test with odds ratio (OR) with $95 \%$ confidence interval $(95 \% \mathrm{CI})$ whenever appropriate. Pearson's correlation test was used to show the correlation of different variables with pruritus intensity. $\mathrm{P}<0.05$ was considered significant.

\section{Results}

In our survey $83 \%$ of psoriatic patients suffered from itching (Table $2 \mathrm{~B}$ ). In $45 \%$ of these patients pruritus appeared daily (in 32\% "often" and in 13\% "always") (Table 2D).

Most patients described pruritus as a sensation of stinging (20\%) and burning (15\%); the intensity reflected by VAS scale was scored as mild only in $13 \%$, moderate in $37 \%$ and severe in $33 \%$ of patients. Seventy-five percent of itch patients had to scratch until bleeding (Table 2C). Itch was most frequent at night, and $50 \%$ of patients reported difficulty in falling asleep.

Pruritus presence and severity was not correlated significantly with patient age, sex, marital status, alcohol and smoking habits, and duration and severity of psoriasis according to Psoriasis Area and Severity Index scores (PASI). ${ }^{6}$ Pruritus intensity and body mass index (BMI) were correlated: $40 \%$ of patients with pruritus were overweight $(\mathrm{BMI}>25<30)$ and $10 \%$ obese $(\mathrm{BMI}>30)$ (Table $2 \mathrm{E})$. Pruritus intensity in these patients was moderate to severe (VAS > 7).

Patients reported regional variations in the sites of pruritus; the most affected anatomical sites were the scalp $(50 \%)$, the lower legs and the back. Some patients reported also a correlation between pruritus and presentation of psoriatic plaques. Itching was otherwise limited to psoriatic lesions (70\% of cases), whereas in $30 \%$ it involved also non-lesional skin.

Stress was identified as an aggravating factor of pruritus by $67 \%$ of the patients. All patients who experienced severe punctual stress before psoriasis onset and exacerbations suffered from pruritus, significantly different compared with subjects in which psoriasis was apparently non-stress-related. ${ }^{7}$

The factors found to reduce itch intensity differed among patients: behavior (such as sunbathing and cold showers), special diets (without nuts, strawberries, red wine, chocolate, blue cheese, milk and sweet products), and specific antipruritic and antipsoriatic treatments.

Among the pruritus treatment modalities, topical therapies such as moisturizers and steroid creams were effective in $24 \%$ and $17 \%$ of patients, respectively, for control of itch in the acute phase but they showed limited long-term effects. Third-generation antihistamines helped for a few days in $13 \%$ of cases. Thirty-six percent of patients did not use any treatment or could not find any effective treatment.

Among the specific antipsoriatic therapies, nb-UVB $\left(311 \mathrm{~nm} ; 2-\mathrm{J} / \mathrm{cm}^{2}\right)$ was shown to be of benefit after a 2 weeks treatment ( 2 or 3 times a week) in 15\% of cases. Biological therapies with a standard dosage of both anti-TNF- $\alpha$ and anti-CD11a relieved pruritus symptoms in the same percentage of patients (Table 2F). In most cases (45\%) no treatment relieved itching.

\section{Conclusions}

All patients enjoyed filling in the questionnaire with the help of a medical doctor, and welcomed the opportunity to talk about their symptoms.

We found a high pruritus prevalence in chronic-plaque type psoriasis vulgaris ( $85 \%$ of patients). Despite a high frequency of this symptom, the pathogenesis of pruritus in psoriasis remains unclear. Cutaneous innervation is high in psoriatic skin with pruritus compared with non-pruritic skin. Sprouting of nerves could accelerate the intensity of itching in inflamed skin of psoriasis patients, ${ }^{8}$ which could be the reason why patients felt more pruritus when the phases of the disease were more active.

Almost all patients agreed that stress is an important enhancing factor for psoriasis and also for pruritus in psoriasis. The patients who did not remember a stress event before the disease onset did not have pruritus. ${ }^{9}$ The mechanism by which stress provokes pruritus in psoriasis is not understood yet; psychological stress causes alterations in levels of certain neuropeptides, such as substance $P$, either in the central nervous system or in tissues. Various mediators as neuropeptides, in addition to histamine released from unmyelinated nerve endings, might have a role. ${ }^{10}$ It is also known that an increase in substance $\mathrm{P}$ potentiates mast cell degranulation in stress condition. Interaction between nerve, neuropeptides and mast cells leads to pruritic inflammation. ${ }^{11}$

Overweight or obese patients experienced more intense pruritus, which could explain the tendency for anatomical folds (some of our psoriatic patients have also previously had inverse psoriasis) to rub together. Moreover, sweating and body temperature increase in obese persons, which worsens pruritus.

Some patients tried to relieve pruritus by taking cold showers. Sensation can be inhibited by substituting the temperature of skin with another sensation to interfere with the steps in the evolution of scratching. Such a substitution may be achieved by cooling the skin by using cold compresses, ice cubes and so forth. 
Table 2 Survey results

A. Information on psoriatic patients involved in the study

B. Psoriatic patients with pruritus (\%)

C. Cluster of intensity of pruritus reflected by VAS scale (\%)

D. Frequency of pruritus in psoriatic patients (\%)

E. Relationship between presence of pruritus and BMI of psoriatic patients (\%)

F. Specific antipsoriatic treatments able to reduce pruritus in psoriatic patients (\%)
$\mathrm{N}=90$

Sex: $45 \mathrm{M} ; 45 \mathrm{~F}$

Age range: $21-84$ years

Disease duration: $1-65$ years

No pruritus $17 \%$

Pruritus 83\%

No pruritus 17\%

Mild I3\%

Moderate 37\%

Severe 33\%

Seldom 55\%

Often 32\%

Always 13\%

Normal weight 50\%

Overweight 40\%

Obese 10\%

None $45 \%$

Topical steroids 8\%

Phototherapy 15\%

Biologicals I5\%

Abbreviations: BMI, body mass index; VAS, visual analog scale.

Currently available drugs to relieve pruritus are not effective and almost all patients were unsatisfied with the available treatment modalities. Various emollients, topical steroids and calcipotriol cream could relieve the pruritus but their effect is temporary. ${ }^{12}$

It is generally accepted that the histamine blockade does not prevent pruritus in psoriasis: $:^{13}$ less than $15 \%$ of psoriatic patients claimed that oral antihistamines were effective in reducing pruritus.

The ineffective action of antihistamines for pruritus in psoriasis might be explained by the fact that various pruritic mediators, separately or combined with histamine, play a role in the mechanism of pruritus in psoriasis vulgaris., ${ }^{9,14}$ Pruritus intensity and histamine plasma level in psoriasis were not correlated, and no difference was observed in histamine plasma levels between pruritic and nonpruritic patients with psoriasis. ${ }^{15}$

Among the antipsoriatic therapies, phototherapy was the most effective treatment effective in reducing pruritus $(15 \%$ of patients on UVB treatment); phototherapy down-regulates substance P-positive nerve fibers, reducing pruritus not only in psoriasis, but also in other inflammatory skin disease. ${ }^{16}$

Both biological therapies, etanercept and efalizumab, were useful in relieving pruritus, in contrast to published data. However, we were not able to establish which of the biologics is more effective, because the number of patients was insufficient to demonstrate statistical significance.
The questionnaire was a useful tool for characterizing itch, and the results might help us to better understand pruritus in psoriasis. The results confirmed the need for a global study of psoriasis with regard to both cutaneous manifestations and the itch symptom.

\section{Disclosures}

The authors have no conflicts of interest to disclose.

\section{References}

1. Bernhard JD. Itch and pruritus: what are they, and how should itches be classified? Dermatol Ther. 2005;18(4):288-291.

2. Reich E, Hrechorow, Szepietowski JC. Negative influence of itching on psoriatic patients' well-being. Acta Dermato-Venereologica. 2007;87(5):478-479.

3. Sampogna F, Gisondi P, Melchi CF, et al. Prevalence of symptoms experienced by patients wuth different clinical types of psoriasis. $\mathrm{BrJ}$ Dermatol. 2004;151:594-599.

4. Yosipovitch G, Goon A, Wee J, Chan YH, Goh CL. The prevalence and clinical characteristics of pruritus among patients with extensive psoriasis. Br J Dermatol. 2000;143:969-973.

5. Yosipovitch G. Assessment of itch: more to be learned and improvements to be made. J Invest Dermatol. 2003;121(6):XIV-XV.

6. Yosipovitch G, Goon A, Wee J, Chan YH, Goh CL. The prevalence and clinical characteristics of pruritus among patients with extensive psoriasis. Br J Dermatol. 2000;143:969-973.

7. Reich A, Szepietowski JC, Wiśnicka B, Pacan P. Does stress influence itching in psoriatic patients? Dermatology and Psychosomatics. 2003;4(3):151-155.

8. Nakamura M, Toyoda M, Morohashi M. Pruritogenic mediators in psoriasis vulgaris: comparative evaluation of itch-associated cutaneous factors. Br J Dermatol. 2003;149:718-730.

9. Gupta MA, Gupta AK, Kirkby S, et al. Pruritus in psoriasis: A prospective study of some psychiatric and dermatologic correlates. Arch Dermatol. 1988;124:1052-1057. 
10. Arck P, Paus R. From the brain-skin connection: the neuroendocrineimmune misalliance of stress and itch. Neuroimmunomodulation. 2007;13(5-6):347-356.

11. Hercogova J. Topical anti-itch therapy. Dermatol Ther. 2005;18: 341-343.

12. Samson YS, Gielczyk R, Scherschun L, Lim HW. Narrow-band ultraviolet B treatment for vitiligo, pruritus and inflammatory dermatoses. Photodermatol Photoimmunol Photomed. 2003;19:164-168.

13. Dawn A, Yosipovitch G. Treating itch in psoriasis. Dermatol Nurs. 2006;18(3):227-233.
14. Reich A, Orda A, Wisnicka B, Szepietowski JC. Plasma neuropeptides and perception of pruritus in psoriasis. J Invest Dermatol. 1989;92: 126-129.

15. Wiśnicka B, Szepietowski JC, Reich A, Orda A. Histamine, substance $P$ and calcitonin gene-related peptide plasma concentration and pruritus in patients suffering from psoriasis. Dermatology and Psychosomatics. 2004;5(2):73-78.

16. Gambichler T, Breuckmann F, Boms S, Altmeyer P, Kreuter A. Narrowband UVB phototherapy in skin conditions beyond psoriasis. J Am Acad Dermatol. 2005;52(4):660-670. 
